\section{CML: TKI sicher für die Niere?}

In einer Studie wurden die Inzidenz akuter Nierenschäden und chronischer Nierenerkrankungen bei Patienten mit chronischer myeloischer Leukämie (CML) unter der Therapie mit Tyrosinkinaseinhibitoren (TKI) untersucht.

$\mathrm{n}$ die Studie aufgenommen wurden 468 Patienten mit neu diagnostizierter CML in der chronischen Phase, die initial einen der TKI Imatinib, Dasatinib oder Nilotinib erhielten. Die mediane Beobachtungsdauer für alle TKI betrug 52 Monate, für Imatinib 87 Monate. $4 \%$ der Patienten hatten in der Nachbeobachtungszeit eine mit TKI assoziierte akute Nierenschädigung - signifikant häufiger unter Imatinib (6\%) als unter Dasatinib oder Nilotinib (1 bzw. $2 \%$; $\mathrm{p}=0,014)$. Im Median trat sie erstmals 9 Tage nach Therapiebeginn auf. Bei 79\% dieser Patienten stieg der Kreatinin-Spiegel um mindestens das 1,5-Fache. Kein
Patient brach die Therapie ab oder wechselte auf einen anderen TKI. Bei 8 von 19 Patienten wurden Faktoren identifiziert, die möglicherweise zur Entwicklung des akuten Nierenschadens beitrugen (Dehydrierung, Diarrhö, gleichzeitige Gabe von Furosemid oder Allopurinol). Die Patienten waren durchschnittlich älter und hatten häufiger Hypertonie, Diabetes mellitus oder eine koronare Herzkrankheit als nicht betroffene Patienten.

$14 \%$ der Patienten entwickelten chronische Nierenerkrankungen unter der Behandlung mit einem TKI, davon $84 \%$ unter Imatinib. Neben der Therapie mit Imatinib waren auch das Alter, Hyperto- nie in der Vorgeschichte und Diabetes mellitus mit einer chronischen Nierenerkrankung assoziiert. Bei Patienten ohne chronische Nierenerkrankung zu Studienbeginn reduzierte Imatinib die glomeruläre Filtrationsrate im Therapieverlauf, interessanterweise aber nicht bei Patienten mit chronischer Nierenerkrankung.

Fazit: TKI können bei CML-Patienten zwar zu akuten Nierenschäden führen, jedoch waren diese meist leicht oder moderat und machten in der Studie keine Änderungen der Therapie erforderlich. Die Ergebnisse deuten außerdem darauf hin, dass TKI bei Patienten mit chronischer Nierenerkrankung ohne Sicherheitsbedenken angewendet werden können. Allerdings sollte ein enges Monitoring gewährleistet sein. Judith Neumaier

Yilmaz M et al. Estimated Glomerular Filtration Rate Changes in Patients With Chronic Myeloid Leukemia Treated With Tyrosine Kinase Inhibitors. Cancer. 2015;121(21):3894-904.

\title{
Fatigue als Prognosefaktor bei MDS?
}

\begin{abstract}
Vom Patienten selbst berichtete Fatigue ist möglicherweise ein wertvoller Prognosefaktor bei schwerem myelodysplastischem Syndrom (MDS).
\end{abstract}

$\mathrm{D}$ er Verlauf eines MDS ist äußerst variabel. Während bei manchen Patienten die Lebenserwartung kaum beeinträchtigt ist, schreitet bei anderen die Erkrankung zur myeloischen Leukämie

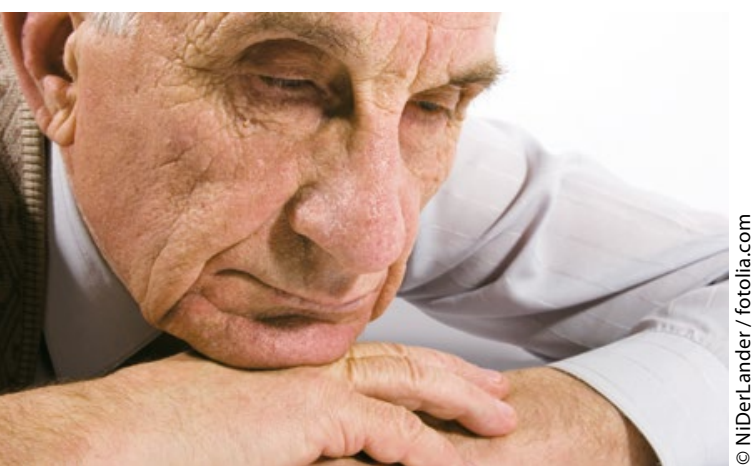

Die Schwere der Fatigue ist bei MDS scheinbar mit dem Überleben assoziiert. fort. Ein Forscherteam untersuchte, inwieweit sich eine Fatigue, über die viele MDS-Patienten klagen, als Prognosefaktor v. a. bei Patienten mit höherem Risiko eignet. Die 280 erwachsenen MDS-Patienten gehörten den IPSS(International Prognostic Scoring System)-Risikogruppen „intermediär 2“ oder „hoch“ an.

Die Fatigue war vor Therapiebeginn mithilfe von Fragen aus dem EORTC QLQ-C30-Fragebogen erfasst worden (Fatigue-Scala: 0-100, höhere Werte sprechen für schwerere Fatigue). Primärer Endpunkt war das Gesamtüberleben. Von den Parametern (u. a. ECOG-Performancestatus, Transfusionspflichtigkeit, Alter und Zahl der Leukozyten), die in der univariaten Analyse mit verkürztem Überleben verknüpft waren, blieben in der multivariaten Analyse nur 2 übrig:

- Bei IPSS-Score ,high“ war das Sterberisiko gegenüber der niedrigsten Kategorie um das 2,5-Fache erhöht (Hazard Ratio [HR] 2,525; $\mathrm{p}=0,0035$ ).

- Auf der Fatigue-Skala war jede Zunahme um 10 Punkte mit einem Risikoanstieg um $11 \%$ assoziiert $(\mathrm{p}=0,0007)$.
Patienten, die 34 oder mehr Punkte auf der Fatigue-Skala aufwiesen, überlebten im Median 14 Monate, wer darunter lag, überlebte im Median $19 \mathrm{Mo}$ nate (HR 1,622; $\mathrm{p}=0,0013)$. Bei 100 Punkten lag die Wahrscheinlichkeit, im ersten Jahr nach der Diagnose zu sterben, bei $56 \%$, bei 0 Punkten bei $20 \%$. Nicht überprüfen lässt sich, ob die Ergebnisse dadurch beeinflusst wurden, dass Patienten mit weniger ausgeprägten FatigueSymptomen möglicherweise eher eine potenziell kurative Therapie erhalten hatten und deshalb länger lebten.

Fazit: Bei neu diagnostizierten Patienten mit MDS und hohem Risiko liefert die selbstberichtete Schwere der Fatigue prognostische Informationen für das Überleben - unabhängig von krankheitsbezogenen Goldstandard-Risikoklassifikationen. Die Forscher empfehlen, Fatigue bei den Patienten im Rahmen der Diagnostik systematisch zu erheben.

Elke Oberhofer

Efficace $\mathrm{F}$ et al. Prognostic value of self-reported fatigue on overall survival in patients with myelodysplastic syndromes: a multicentre, prospective, observational, cohort study. Lancet Oncol. 2015;16(15):1506-14. 